\title{
INDICADORES DE DESEMPENHO COMO FERRAMENTA DE ALINHAMENTO ESTRATÉGICO - A EXPERIÊNCIA DO SENAC PARANÁ
}

\section{RESUMO}

Este artigo apresenta um estudo de caso de apropriação dos conceitos do Balanced Scorecard pelo Serviço Nacional de Aprendizagem Comercial (SENAC) do Paraná, por meio da elaboração de indicadores de desempenho para alinhamento estratégico. A execução da diretriz estratégica de promoção social, um dos pilares da instituição, foi medida por meio de indicadores que visam acompanhar as ações de gratuidade das unidades, sob a premissa de que elas dão sustentação à referida estratégia. Os indicadores formulados têm enfoque tanto na produtividade quanto no cumprimento das metas de gratuidade estabelecidas, e foram submetidos a estudo estatístico por meio da técnica de análise de variância, cujo objetivo é evidenciar a existência de diferença significativa no rol de dados analisados. $\mathrm{O}$ resultado aponta que, de fato, há diferença no desempenho das unidades avaliadas, razão pela qual são sugeridos questionamentos com o intuito de auxiliar o processo de alinhamento das estratégias locais à estratégia global, com o objetivo de otimizar a oferta de cursos gratuitos em todas as regiões do Estado. O presente estudo de caso relata a experiência do SENAC Paraná, com escopo de informações limitado ao ano de 2013 e relativo às 33 escolas de educação profissional da instituição, distribuídas em todo o Estado.

Palavras-chave: Balanced Scorecard; Indicadores De Desempenho; Gratuidade; Senac; Alinhamento Estratégico.

\section{PERFORMANCE INDICATORS AS A STRATEGIC ALIGNMENT TOOL - THE EXPERIENCE OF SENAC PARANÁ}

\begin{abstract}
This article presents a case study of the appropriation of Balanced Scorecard's concepts by SENAC Paraná, through the development of performance indicators for strategic alignment. The implementation of the strategic direction of social promotion, one of the pillars of the institution, was measured through indicators that aim to monitor the units' actions of gratuitousness, under the premise that they sustain this strategy. The focus of the indicators is both productivity and meeting the targets of gratuity. These indicators were subjected to statistical analysis by the technique of analysis of variance, whose goal is to show the existence of significant differences in the list of data analyzed. The result shows that, in fact, there is difference in the performance of the units, which is why questions are suggested in order to assist the process of alignment between local and global strategies, with the objective of optimizing the supply of free courses in all regions of the state. This case study describes the experience of SENAC Paraná, with scope limited to the year 2013 and on the 33 schools for vocational education institution, distributed throughout the state information.
\end{abstract}

Keywords: Balanced Scorecard; Performance Indicators; Gratuitousness; Senac; Strategy Alignment. 
INDICADORES DE DESEMPEÑO COMO HERRAMIENTA ESTRATÉGICA ALINEACIÓN - EL SENAC EXPERIENCIA PARANÁ

\section{RESUMEN}

En este artículo se presenta un estudio de caso de la apropiación de los conceptos del Balanced Scorecard por el Servicio Nacional de Aprendizaje Comercial (SENAC) de Paraná, a través del desarrollo de indicadores de desempeño para la alineación estratégica. La aplicación de la política estratégica de la promoción social, uno de los pilares de la institución, se midió a través de indicadores que apuntan a monitorear las acciones de la gratuidad de las unidades bajo la premisa de que dan soporte a esa estrategia. Los indicadores formulados tienen centrarse tanto en la productividad y en el cumplimiento de las metas establecidas gratuidad y se sometieron a análisis estadístico mediante un análisis técnico de la varianza, que tiene como objetivo demostrar la existencia de diferencias significativas en la lista de los datos analizados. El resultado indica que, de hecho, no hay diferencia en el desempeño de las unidades evaluadas, por lo que las preguntas se sugieren para ayudar a la alineación de las estrategias locales a la estrategia global, con el objetivo de optimizar la prestación de cursos gratuitos en todo regiones del estado. Este estudio de caso se presenta la experiencia Senac Paraná, con información de alcance limitado para el año 2013 y en las 33 escuelas de formación profesional de la institución, distribuidos en todo el estado.

Palabras clave: Cuadro de Mando Integral; En los Indicadores de Rendimiento; La gratuidad; Senac; Alineamiento Estratégico.

Solan Arantes Valente ${ }^{1}$

\footnotetext{
${ }^{1}$ Mestrando em Ciência, Gestão e Tecnologia da Informaçãopela Universidade Federal do Paraná - UFPR. Atua como assessor da direção regional do SENAC Paraná, na área de estratégia e negócios. Brasil. E-mail: solanvalente@gmail.com
} 


\section{INTRODUÇÃO}

O BSC (Balanced Scorecard) é um dos mais difundidos modelos dentre os sistemas de medição de desempenho da atualidade. Em sua essência, preconiza a formulação de indicadores de desempenho como ferramenta para acompanhar a materialização da estratégia organizacional, no âmbito operacional. Os indicadores medem, de forma sistêmica, a viabilização das estratégias globais, alinhando-as às iniciativas locais e promovendo uma evolução do desempenho ao longo do tempo.

As diretrizes estratégicas são referenciais para a atuação de qualquer instituição, e devem estar alinhadas à sua missão e visão, observando a cultura organizacional, bem como seus valores e crenças. Caiuby e Luciano (2005, p. 66) destacam que o alinhamento dos processos com a estratégia é essencial para o desempenho da organização.

Kaplan e Norton (2001, p.175) salientam a importância do alinhamento organizacional para criar sinergias, induzindo à consciência acerca da necessidade de se gerir a "grande estratégia" corporativa, com o intuito de integrar vários negócios e unidades de apoio.

Segundo Niven (2003, p. 23, p. 33), as diretrizes estratégicas estabelecem as grandes prioridades que a organização elenca para atingir sua missão, a partir de um determinado ambiente. A estratégia evidencia o "como fazer" para alcançar as metas e objetivos almejados. Para o autor, o principal papel do BSC é promover a tradução e oportunizar o equilíbrio da estratégia. Para Gaio (2012, p. 53), tal tradução pode ocorrer quando a missão é decodificada em valores, visão, objetivos, iniciativas e medidas de desempenho de maneira coerente, garantindo que todos os colaboradores tenham acesso às informações para direcionarem seus esforços.

O BSC traduz a missão e a estratégia das empresas num conjunto abrangente de medidas de desempenho que serve de base para um sistema de medição e gestão estratégica. (KAPLAN, NORTON, 1997, p. 2). A componente "balanced" que nomeia a metodologia sugere equilíbrio; desta forma, o BSC mede o desempenho organizacional sob quatro perspectivas equilibradas: financeira, dos clientes, dos processos internos da empresa e do aprendizado e crescimento. (KAPLAN, NORTON, 1997, p. 2).

Para Lima, Soares e Lima (2011), verifica-se nas organizações, de maneira geral, uma tendência em relação aos sistemas de avaliação de desempenho serem reduzidos à dimensão financeira. Para os autores, as instituições de ensino superior, em geral, não fogem à regra, atribuindo peso maior a aspectos financeiros na formulação e utilização dos indicadores estratégicos.

Se as diretrizes estratégicas devem ser coerentes com a razão da existência da organização, muitas empresas falham no processo de alinhamento das iniciativas e projetos às estratégias inicialmente formuladas, cuja finalidade primordial é assegurar que a missão institucional seja de fato realizada. Sem instrumentos que garantam tal alinhamento, oportunizando aos gestores tanto mensuração quanto controle, muitas iniciativas emanadas das unidades de negócios, embora bem intencionadas, tendem a resumir-se em dispêndios de recursos que pouco agregam às diretivas estratégicas e ao cumprimento da missão organizacional.

Damke, Silva e Walter (2011) afirmam que a utilização de sistemas de controle estratégico que garantam, por meio do monitoramento dos indicadores, a manutenção das organizações na direção estratégica previamente definida, não é uma tarefa simples. Para os autores, os indicadores devem ter a finalidade de evidenciar a eficiência dos processos organizacionais e, se não forem corretamente formulados, podem levar a resultados que não condizem com a realidade, comprometendo a tomada de decisão.

Wunder (2005, p. 39) trabalha o conceito de alinhamento vertical, referindo-se à tentativa de se assegurar que as ações nos níveis mais baixos, a partir das unidades de negócio, devem acontecer em paralelo à corporação e alinhadas ao seu direcionamento estratégico.

Segundo Kaplan e Norton, este não é um processo simples. Para os autores,

O alinhamento da organização a uma visão compartilhada e uma direção comum é um processo demorado e complexo. As grandes organizações adotam vários mecanismos integrados para traduzir a estratégia e o balanced scorecard em objetivos e medidas locais que influenciem as prioridades das pessoas e das equipes. (KAPLAN, NORTON, 1997, p. 210)

Segundo os autores, está posto um dos grandes desafios neste processo: a tradução da estratégia global em objetivos e medidas reconhecidas pelas equipes executoras. Neste contexto, o BSC surge como ferramenta que permite o compartilhamento da direção comum e a materialização desta em metas tangíveis.

Para Johnston e Pongatichat (2008, p. 19), é evidente a escassez de modelos e métodos robustos que auxiliem na execução da estratégia e oportunizem a mensuração e o alinhamento estratégico. Com isso, a estratégia da unidade de negócios passa a refletir a percepção individual do gestor acerca das ações que, na sua visão, seriam as mais acertadas e oportunas em dadas circunstâncias, e de cuja percepção derivam as iniciativas. Está caracterizado assim, em sua plenitude, o desalinhamento estratégico das ações do nível operacional, perpetuando-se nas unidades de negócios as mais diferentes formas de atuação, variando na mesma proporção dos estilos de gestão. Nesse sentido, Muller et al (2003) salientam que um modelo 
equilibrado para avaliação do desempenho deve incorporar, na formulação dos indicadores, aspectos inerentes às formas de controle empregadas na organização, bem como a sua relação com o planejamento estratégico, com os estilos de gestão presentes e com a cultura organizacional.

Achwarz, Amiden e Pinho (2005, p. 17, p. 23) descrevem que o processo de desdobramento da estratégia acarreta na necessidade de se definir objetivos, indicadores, metas e iniciativas, imersas na condição estratégica suigeneris de cada unidade de negócio, e como esta singularidade contribui para a estratégia corporativa. Tal desdobramento da estratégia, segundo os autores, está vinculado à sua tradução; salientando, ainda, que cabe aos gestores analisar qual é a melhor forma de organizar a estrutura para executar a estratégia, uma vez que a estrutura disponível e vigente pode não garantir sua correta execução.

Para Costa (2006, p. 11) o BSC trata das informações-chaves para subsidiar o gerenciamento do cumprimento da estratégia da organização e dos processos gerenciais, que devem ser aptos a promover o alinhamento estratégico necessário. Entretanto, processos envolvem pessoas e, nesse sentido, Kaplan e Norton afirmam que:

A implementação da estratégia começa pela capacidade e envolvimento das pessoas que devem executá-la ... o ideal seria que todos na empresa, do nível hierárquico mais elevado ao mais baixo, compreendessem a estratégia e como as suas ações individuais sustentam o "quadro geral". O BSC permite este alinhamento de cima para baixo. (KAPLAN, NORTON, 1997, p. 208)

Com o intuito de sistematizar o monitoramento deste alinhamento, o modelo BSC propõe a criação de indicadores de desempenho, que são medidas cuja finalidade é evidenciar a aderência das iniciativas locais às estratégias globais. Tais indicadores tornam tangível a materialização da estratégia, fornecendo aos gestores números que promovem uma fácil leitura do desempenho das unidades de negócios, sob a forma de índices cuidadosamente elaborados para inter-relacionarem informações que traduzem, em sua essência, quanto da estratégia tem permeado as iniciativas.

Para Fernandes e Berton,

O BSC procura desenvolver um senso comum no processo de gestão, definindo seus critérios em variáveis fundamentais para a perfeita harmonia entre os setores e níveis decisórios da empresa, e facilitar o processo de comunicação entre esses níveis, por meio do esclarecimento e tradução da missão e visão da empresa, bem como da estratégia adotada. (FERNANDES, BERTON, 2004, p. 207)
Nesse sentido, o Serviço Nacional de Aprendizagem Comercial (SENAC) do Paraná desenvolveu uma metodologia que se apropria dos conceitos do BSC para aferir o desempenho das unidades de negócios, com o intuito de evidenciar possíveis distorções em relação às principais diretivas estratégicas da instituição. Embora trate-se de uma ferramenta utilizada em larga escala por instituições privadas que visam lucro, o estudo de Herman e Renz (1999) evidencia que muitas práticas incorporadas pelas organizações sem fins lucrativos (OSFL) provém da realidade empresarial, como planejamento estratégico, eficácia e gestão de qualidade, todavia sem a reflexão requerida e necessária sobre as implicações destas práticas em seu contexto diferenciado. Um dos objetivos deste artigo, portanto, é auxiliar no processo de utilização do BSC por empresas de economia mista, sem fins lucrativos, em especial por aquelas inseridas nos Serviços Nacionais de Aprendizagem (conhecidos como Sistema S), com a devida reflexão em relação ao seu campo de atuação e à sua missão, apropriando-se da ferramenta para oportunizar o alinhamento das estratégias primordiais da instituição às iniciativas locais.

Por se tratar de uma organização com figura jurídica mista, permeada por características tanto de instituição privada quanto pública, o SENAC Paraná tem buscado permanentemente ferramentas que viabilizem o controle dos processos internos com vistas ao atendimento às auditorias dos órgãos de controle e às exigências legais impostas pelo Governo, conciliando esta realidade à necessidade de sustentabilidade econômico-financeira, condição sine qua non à sua perenidade e expansão. Neste contexto de dualidade onde encontram-se inseridas grande parte das OSFL, segundo Gaio:

É comum que diversos $r$ atores
governamentais, financiadores e a própria
sociedade passem a cobrar das OSFL o
desempenho de resultado e a transparência. O
aumento da competitividade por recursos tem
intensificado essas organizações a adotar
mecanismos de gestão r mais
profissionalizados, assemelhando-se às
organizações empresariais. (GAIO, 2012, p.
26)

O viés público que exerce influência na atuação do SENAC diz respeito ao recolhimento de contribuição para-fiscal compulsória dos empresários do comércio de bens, serviços e turismo, o que confere à instituição um caráter sindical patronal. Esta contribuição é regulada pelo Decreto Lei $\mathrm{n}^{\circ}$ 8.621, de 1946, que instituiu a criação do Serviço Nacional de Aprendizagem Comercial. Tal realidade remete o SENAC a auditorias de contas organizadas por órgãos de controle externos, como CGU (Controladoria Geral da União) e TCU (Tribunal de Contas da União), e internos, como Conselho Fiscal e empresas licitadas 
pela instituição com esta finalidade. Ressalta-se, nesse contexto, o enquadramento da instituição na Lei $\mathrm{n}^{\circ}$ 8.666, de 1993, que regula os processos licitatórios, bem como na Lei $n^{\circ} 12.527$, de 2011, que estabelece critérios que garantam total transparência na utilização de recursos públicos.

A partir de 2009, após Protocolo de Compromisso firmado com o Governo Federal, foi instituído o PSG (Programa SENAC de Gratuidade), que compromete, de forma gradativa ao longo dos anos, o recurso da contribuição compulsória na oferta de cursos gratuitos de qualificação profissional à população de baixa renda. Os percentuais de aplicação da receita advinda da contribuição para-fiscal compulsória do empresariado em cursos gratuitos evoluíram da seguinte forma: $20 \%$ em $2009 ; 25 \%$ em 2010; 35\% em 2011; $45 \%$ em 2012; 55\% em 2013 e, finalmente, 66,6\% em 2014. Tal aplicação, decorrente de exigência legal, tem demandado da instituição, desde sua idealização, a busca permanente por instrumentos e ferramentas que garantam a gestão de todo este processo, com vistas ao pleno atendimento ao compromisso acordado. Ao mesmo tempo, tem impulsionado a instituição a incrementar sua receita própria, decorrente da venda de cursos no varejo, com vistas à sua sustentabilidade financeira e à materialização do seu plano de expansão.

Segundo Walter et al (2013), "as mudanças que vêm ocorrendo no mercado impactaram não apenas empresas produtivas e de serviços, privadas ou públicas, mas também, de forma importante, entidades que atuam no terceiro setor". Manzione (2006) salienta que o terceiro setor tem crescido no Brasil por meio de entidades de dimensões variadas que, embora não tenham fins lucrativos, necessitam cada vez mais desenvolver estratégias que garantam qualidade dos serviços, competitividade e sobrevivência no mercado.

A Lei $n^{\circ}$ 12.513, de 2011, que instituiu o PRONATEC (Programa Nacional de Acesso ao Ensino Técnico e Emprego), delegou aos Serviços Nacionais de Aprendizagem (Sistema S) a condição de principais executores do programa. Tal enquadramento consolida ao SENAC a condição de um dos principais agentes na execução de políticas públicas de educação profissionalizante, protagonizando assim notório processo de descentralização da administração pública. A escolha pelos Serviços Nacionais de Aprendizagem, balizada em sua capilaridade, transparência e qualidade de ensino reconhecidas, confere ao Sistema $\mathrm{S}$ a condição ainda mais veemente de prestador de serviços públicos, confiando a ele a gestão de recursos oriundos do Governo Federal que, em conjunto com os recursos advindos da contribuição do empresariado, dão sustentação e vazão à oferta dos dois grandes programas de gratuidade do Sistema.

Dado este cenário de atuação, permeado de forma marcante pelos vieses público e político, evidencia-se a necessidade de controle permanente tanto de processos, em função de possíveis apontamentos em auditorias internas e externas, quanto de alinhamento das principais estratégias institucionais no âmbito operacional das unidades executivas do SENAC. Desta forma, o controle da execução da gratuidade, que é objeto de estudo deste artigo, ganha importância estratégica no cenário ora descrito.

\section{OBJETIVOS}

$\mathrm{O}$ presente artigo tem como objetivo geral descrever a experiência do SENAC na apropriação dos conceitos do BSC, bem como a forma utilizada para criação e análise estatística de indicadores de desempenho com foco na execução dos programas de gratuidade, acompanhando desta forma a materialização da diretiva estratégica de promoção social.

Como objetivo específico, tem-se a aferição do êxito na aplicação dos programas de gratuidade, que é mensurado a partir de dois indicadores: de produtividade e de cumprimento de metas. $\mathrm{O}$ indicador de produtividade proposto abarca informações de quantitativo de pessoal em relação ao total de investimentos em gratuidade, em cada uma das unidades. Já o indicador de cumprimento de metas propõe relacionar os valores investidos em gratuidade com os valores previamente orçados por cada gestor, em cada unidade executiva.

Após a geração dos indicadores, é então aplicada sobre as informações a técnica estatística de análise de variância, com o propósito de evidenciar diferenças significativas nos índices, em cada uma das unidades. Uma vez identificadas as não conformidades, sugerem-se questionamentos para identificação das melhores práticas, com vistas ao alinhamento da execução da estratégia em todos os pontos de atendimento.

\section{JUSTIFICATIVA}

O alinhamento estratégico promove o uso racional e otimizado dos recursos em prol da missão organizacional.

Dentre as cinco diretivas estratégicas do SENAC Paraná, a saber: orientação para o mercado; inovação e gestão do conhecimento; gestão institucional; imagem institucional e promoção social, esta última foi eleita, no estudo de caso em questão, pela sua importância no contexto de atuação da instituição. Enquanto agente executor de políticas públicas de educação profissional e, por conseguinte, de inclusão produtiva da população de baixa renda, verificar se tal diretiva está sendo de fato materializada traz, a toda a sociedade, ganhos imensuráveis. 


\section{METODOLOGIA}

Tendo como balizadora a diretiva estratégica de promoção social, o estudo de caso em questão apresenta a forma utilizada pelo SENAC Paraná para acompanhamento da sua materialização, no âmbito das suas 33 unidades executivas, mediante a formulação de indicadores de desempenho, com foco na execução dos programas de gratuidade, de dois tipos:

1. Indicador de produtividade: com foco na efetividade da mão de obra das escolas em análise, em relação à gratuidade ofertada no período em questão.

Este indicador considera o montante de investimentos executados nos dois grandes programas de gratuidade (PSG e PRONATEC), no ano de 2013, em relação ao número total de colaboradores efetivos, em cada uma das unidades executivas.

Tal indicador reflete comparativamente $\mathrm{o}$ nível de produtividade/homem na execução da gratuidade, em todas as unidades, e é indicado para se comparar escolas sob uma distribuição isonômica de verbas para execução dos programas em questão. Nesse caso, não há favorecimento de uma unidade em detrimento de outra, por questões estratégicas de direcionamento de cotas extras para gratuidade em função de cenários socioeconômicos ou políticos.

$\mathrm{O}$ indicador é gerado somando-se os valores totais investidos no PSG e no PRONATEC, em cada unidade executiva, nos quatro trimestres do ano em análise. Em seguida, este valor é dividido pelo número total de colaboradores efetivos do quadro administrativo das unidades. $\mathrm{O}$ índice mostra, desta forma, qual o valor investido em relação à força de trabalho disponível, evidenciando a efetividade na utilização dos recursos humanos em prol da aplicação dos programas de gratuidade. Os resultados obtidos podem ser observados na Tabela 1.

2. Indicador de metas: com foco nos investimentos em gratuidade realizados em relação ao montante orçado para o período em questão, em cada uma das unidades.

Em muitos casos, não é estratégico aplicar a gratuidade na mesma proporção, em todas as regiões. Esta questão é observada no processo de dimensionamento das metas de gratuidade em cada uma das regiões, abarcando em sua discussão elementos como IDH (índice de desenvolvimento humano) médio. Níveis mais baixos deste índice pressupõem a necessidade de maiores investimentos em educação e aplicação, por conseguinte, de gratuidade. Tal estratégia visa assegurar o papel do SENAC enquanto promotor de desenvolvimento humano por meio da inclusão produtiva.

Quando tal situação ocorrer, a prática proposta é a geração de indicadores que reflitam os percentuais de aplicação de gratuidade em relação às metas, que trazem em seu dimensionamento toda a problemática socioeconômica e política descrita. Aplica-se, então, a mesma técnica estatística a esta massa de dados, permitindo-se comparar unidades não mais em relação à produtividade, mas em relação ao percentual da meta proposta de aplicação de gratuidade efetivamente atingida.

O indicador é gerado somando-se os valores totais investidos no PSG e no PRONATEC, em cada unidade executiva, nos quatro trimestres do ano em análise. Em seguida, este valor é dividido pelo valor orçado para investimento em cada trimestre, representando, em termos percentuais, a parcela da meta (ou valor orçado) que foi efetivamente executada em cada unidade. Os valores obtidos podem ser observados na Tabela 3 .

Após a formulação e tabulação dos indicadores, é aplicada sobre os dados a técnica estatística de análise de variância, cuja finalidade é estabelecer se há diferença significativa entre os indicadores gerados, nas diferentes unidades de educação profissionalizante analisadas. Os dados foram analisados com o auxílio do software estatístico BIOESTAT, de domínio público, disponível no endereço eletrônico http://www.mamiraua.org.br/ptbr/downloads.

Uma vez verificada a existência de diferença significativa entre a performance de diferentes unidades, no mesmo indicador, são propostos questionamentos com o objetivo de se aferir as estratégias e práticas adotadas pelas unidades com melhor desempenho nos indicadores em análise. Tais questionamentos têm a missão de iniciar o processo corretivo, promovendo o alinhamento das ações à diretiva estratégica institucional.

\section{RESULTADOS}

A Tabela 1 apresenta os resultados obtidos no indicador de produtividade, mostrando a aplicação total de gratuidade executada por colaborador do quadro efetivo de cada unidade, no ano de 2013. A metodologia para sua formulação foi previamente descrita no capítulo anterior.

Nesta análise foram consideradas comparativamente apenas unidades cujas cotas de gratuidade seguem uma distribuição isonômica, proporcional à dimensão das mesmas em relação à produção e à estrutura de custos. Desta forma, foram desconsideradas unidades com distribuição de cotas extras, no exercício de 2013, oriundas de diretivas de distribuição de gratuidade decorrentes de demandas socioeconômicas ou políticas. 
Indicadores de Desempenho como Ferramenta de Alinhamento Estratégico - A Experiência do Senac Paraná

Tabela 1 - Gratuidade média aplicada por colaborador efetivo do quadro em 2013.

\begin{tabular}{|c|c|c|c|c|c|c|c|}
\hline \multirow{3}{*}{$\begin{array}{l}\text { UNIDADE } \\
\text { UEP1 }\end{array}$} & \multicolumn{7}{|c|}{ GRATUIDADE MÉDIA EXECUTADA POR COLABORADOR - 2013} \\
\hline & $1^{\circ}$ Trimestre & \multicolumn{2}{|c|}{$2^{\circ}$ Trimestre } & \multicolumn{2}{|c|}{$3^{\circ}$ Trimestre } & \multicolumn{2}{|c|}{$4^{\circ}$ Trimestre } \\
\hline & $\mathrm{R} \$ 22.042,21$ & & $31.463,05$ & $\mathrm{R} \$$ & $33.059,55$ & $\mathrm{R} \$$ & $19.853,06$ \\
\hline UEP2 & $\mathrm{R} \$ \quad 10.362,48$ & $\mathrm{R} \$$ & $12.354,48$ & $\mathrm{R} \$$ & $39.145,44$ & $\mathrm{R} \$$ & $69.022,54$ \\
\hline UEP5 & $\mathrm{R} \$ 58.401,07$ & $\mathrm{R} \$$ & $64.610,99$ & $\mathrm{R} \$$ & $73.518,82$ & $\mathrm{R} \$$ & $67.484,98$ \\
\hline UEP6 & $\mathrm{R} \$ 31.712,20$ & $\mathrm{R} \$$ & $43.186,31$ & $\mathrm{R} \$$ & $44.078,15$ & $\mathrm{R} \$$ & $35.245,44$ \\
\hline UEP7 & $\mathrm{R} \$ \quad 49.147,80$ & $\mathrm{R} \$$ & $58.740,53$ & $\mathrm{R} \$$ & $90.361,59$ & $\mathrm{R} \$$ & $53.484,66$ \\
\hline UEP8 & $\mathrm{R} \$ \quad 20.575,89$ & $\mathrm{R} \$$ & $35.401,01$ & $\mathrm{R} \$$ & $35.673,95$ & $\mathrm{R} \$$ & $26.580,86$ \\
\hline UEP9 & $\mathrm{R} \$ \quad 23.408,41$ & $\mathrm{R} \$$ & $42.154,68$ & $\mathrm{R} \$$ & $52.353,96$ & $\mathrm{R} \$$ & $32.831,86$ \\
\hline UEP10 & $\mathrm{R} \$ \quad 40.424,36$ & $\mathrm{R} \$$ & $48.652,98$ & $\mathrm{R} \$$ & $45.341,79$ & $\mathrm{R} \$$ & $50.495,50$ \\
\hline UEP11 & $\mathrm{R} \$ \quad 42.130,70$ & $\mathrm{R} \$$ & $54.969,76$ & $\mathrm{R} \$$ & $41.497,40$ & $\mathrm{R} \$$ & $48.565,18$ \\
\hline UEP12 & $\mathrm{R} \$ \quad 9.076,22$ & $\mathrm{R} \$$ & $14.752,69$ & $\mathrm{R} \$$ & $33.287,87$ & $\mathrm{R} \$$ & $40.072,56$ \\
\hline UEP13 & $\mathrm{R} \$ \quad 45.747,00$ & $\mathrm{R} \$$ & $75.465,15$ & $\mathrm{R} \$$ & $76.522,65$ & $\mathrm{R} \$$ & $54.372,93$ \\
\hline UEP14 & $\mathrm{R} \$ 26.923,76$ & $\mathrm{R} \$$ & $45.189,78$ & $\mathrm{R} \$$ & $50.933,35$ & $\mathrm{R} \$$ & $30.317,59$ \\
\hline UEP15 & $\mathrm{R} \$ 27.614,64$ & $\mathrm{R} \$$ & $41.943,51$ & $\mathrm{R} \$$ & $50.162,43$ & $\mathrm{R} \$$ & $44.602,10$ \\
\hline UEP17 & $\mathrm{R} \$ \quad 42.379,40$ & $\mathrm{R} \$$ & $64.634,47$ & $\mathrm{R} \$$ & $61.706,72$ & $\mathrm{R} \$$ & $49.878,06$ \\
\hline UEP18 & $\mathrm{R} \$ 21.436,36$ & $\mathrm{R} \$$ & $35.114,24$ & $\mathrm{R} \$$ & $44.525,28$ & $\mathrm{R} \$$ & $27.772,63$ \\
\hline UEP19 & $\mathrm{R} \$ \quad 54.315,42$ & $\mathrm{R} \$$ & $64.993,37$ & $\mathrm{R} \$$ & $62.379,98$ & $\mathrm{R} \$$ & $53.483,28$ \\
\hline UEP20 & $\mathrm{R} \$ 34.098,11$ & $\mathrm{R} \$$ & $47.162,31$ & $\mathrm{R} \$$ & $47.861,30$ & $\mathrm{R} \$$ & $27.264,23$ \\
\hline UEP22 & $\mathrm{R} \$ 12.341,96$ & $\mathrm{R} \$$ & $26.170,52$ & $\mathrm{R} \$$ & $32.106,98$ & $\mathrm{R} \$$ & $46.149,70$ \\
\hline UEP23 & $\mathrm{R} \$ \quad 3.104,20$ & $\mathrm{R} \$$ & $73.940,55$ & $\mathrm{R} \$$ & $86.039,40$ & $\mathrm{R} \$$ & $71.222,83$ \\
\hline UEP24 & $\mathrm{R} \$ \quad 16.848,00$ & $\mathrm{R} \$$ & $29.970,30$ & $\mathrm{R} \$$ & $64.137,10$ & $\mathrm{R} \$$ & $133.831,16$ \\
\hline UEP25 & $\mathrm{R} \$ 70.260,91$ & $\mathrm{R} \$$ & $68.579,20$ & $\mathrm{R} \$$ & $35.969,83$ & $\mathrm{R} \$$ & $67.161,95$ \\
\hline UEP26 & $\mathrm{R} \$ \quad 46.510,43$ & $\mathrm{R} \$$ & $57.065,95$ & $\mathrm{R} \$$ & $82.182,63$ & $\mathrm{R} \$$ & $112.237,74$ \\
\hline UEP27 & $\mathrm{R} \$ 31.293,33$ & $\mathrm{R} \$$ & $45.776,83$ & $\mathrm{R} \$$ & $59.597,68$ & $\mathrm{R} \$$ & $69.612,10$ \\
\hline UEP28 & $\mathrm{R} \$ 38.827,00$ & $\mathrm{R} \$$ & $38.827,00$ & $\mathrm{R} \$$ & $48.048,00$ & $\mathrm{R} \$$ & $105.500,16$ \\
\hline UEP29 & $\mathrm{R} \$ \quad 11.354,40$ & $\mathrm{R} \$$ & $19.693,13$ & $\mathrm{R} \$$ & $26.424,87$ & $\mathrm{R} \$$ & $27.939,87$ \\
\hline UEP30 & $\mathrm{R} \$ 27.095,10$ & $\mathrm{R} \$$ & $113.603,93$ & $\mathrm{R} \$$ & $114.835,80$ & $\mathrm{R} \$$ & $116.335,90$ \\
\hline UEP32 & $\mathrm{R} \$ 38.093,52$ & $\mathrm{R} \$$ & $44.299,15$ & $\mathrm{R} \$$ & $47.802,51$ & $\mathrm{R} \$$ & $58.695,10$ \\
\hline UEP33 & $\mathrm{R} \$ \quad 16.852,41$ & $\mathrm{R} \$$ & $30.700,83$ & $\mathrm{R} \$$ & $38.591,88$ & $\mathrm{R} \$$ & $13.394,38$ \\
\hline
\end{tabular}

Fontes: Relatórios dos sistemas gerenciais Sapiens e Rubi - ano de 2013.

Estabelecendo-se como hipótese nula (Ho) que o montante total de investimentos em gratuidade por colaborador independe da unidade, aplica-se então a técnica estatística de análise de variância, cuja finalidade é evidenciar se há diferença significativa no rol de dados apresentados.

O resultado da análise estatística dos dados da Tabela 1 pode ser observado na Tabela 2 :

Tabela 2 - Análise estatística da gratuidade média executada por colaborador em 2013

\begin{tabular}{|l|c|c|c|c|}
\hline & $\mathbf{1}^{\mathbf{0}}$ Trimestre & $\mathbf{2}^{\mathbf{o}}$ Trimestre & $\mathbf{3}^{\mathbf{o}}$ Trimestre & $\mathbf{4}^{\mathbf{o}}$ Trimestre \\
\hline Tamanho da amostra & 28 & 28 & 28 & 28 \\
\hline Média Aritmética & 31156.3318 & 47479.1679 & 54219.5325 & 55478.8696 \\
\hline Variância & $26989 \mathrm{e}+04$ & $45618 \mathrm{e}+04$ & $43624 \mathrm{e}+04$ & $91841 \mathrm{e}+04$ \\
\hline Desvio Padrão & 16428.3971 & 21358.2869 & 20886.4650 & 30305.3203 \\
\hline Valor da estatística F: 6,7327; nível de significância: 5\%.
\end{tabular}


Desta forma, rejeita-se a hipótese nula $(\mathrm{Ho}) \mathrm{e}$ aponta-se para a existência de diferença estatisticamente significativa na média de gratuidade aplicada por colaborador, nas unidades analisadas, ao longo do ano de 2013.

A análise de variância é aqui apresentada como uma técnica estatística aplicada à gestão. Uma vez verificado que o valor da estatística F de Snedecor, na massa de dados analisada, é superior aos valores críticos da tabela, ao nível de significância dado, fica comprovada a existência de diferença estatisticamente relevante nos valores, inferindo-se assim a necessidade de uma análise mais aprofundada das possíveis causas.

Dada esta situação, os questionamentos sugeridos após a análise do indicador "gratuidade média por colaborador" são os seguintes:

- Quais as estratégias adotadas pelos gestores das unidades com melhor performance?

- Que modalidades de cursos foram ofertadas nestas unidades?

- Como está ocorrendo a oferta em unidades remotas, cedidas por instituições parceiras?

- Como se dá o processo de divulgação dos programas nestas unidades?

- Quais as práticas adotadas pelos gestores das unidades destaque, voltadas ao engajamento das equipes?
Tais questionamentos podem auxiliar na identificação das melhores práticas, que têm resultado no melhor desempenho de algumas unidades, conforme evidenciado na Tabela 1.

A Tabela 3 apresenta o indicador que reflete o percentual de execução dos investimentos orçados em gratuidade, somando-se as metas do PSG e do PRONATEC, por unidade, no ano de 2013. A metodologia para sua formulação foi previamente descrita no capítulo anterior.

Esta abordagem permite a comparação de todas as unidades, independentemente de sua dimensão ou do direcionamento de cotas extras de gratuidade, uma vez que relativiza os resultados às metas propostas pelos próprios gestores. Tais metas são o produto de uma análise prévia da capacidade e do histórico de execução de cada unidade, lançados em um orçamento programa que é monitorado mensalmente e retificado no meio do exercício (no mês de julho), para que possa ser alinhado ao processo real de execução.

Desta forma, o percentual de meta atingida, evidenciado por este indicador, compara a efetividade de cada escola na execução da gratuidade proposta para aquele exercício.

Tabela 3 - Percentual de execução do investimento em gratuidade orçado para 2013.

\begin{tabular}{|l|c|c|c|c|}
\hline \multirow{2}{*}{ UNIDADE } & \multicolumn{5}{|c|}{$\%$ DA META DE GRATUIDADE ATINGIDA - 2013 } \\
\cline { 2 - 5 } & $\mathbf{1}^{\mathbf{0}}$ Trimestre & $\mathbf{2}^{\mathbf{0}}$ Trimestre & $\mathbf{3}^{\mathbf{0}}$ Trimestre & $\mathbf{4}^{\mathbf{0}}$ Trimestre \\
\hline \multirow{2}{*}{ UEP1 } & $87 \%$ & $105 \%$ & $110 \%$ & $80 \%$ \\
\hline UEP2 & $46 \%$ & $48 \%$ & $153 \%$ & $323 \%$ \\
\hline UEP4 & $87 \%$ & $135 \%$ & $131 \%$ & $80 \%$ \\
\hline UEP5 & $238 \%$ & $132 \%$ & $330 \%$ & $-59 \%$ \\
\hline UEP6 & $125 \%$ & $121 \%$ & $138 \%$ & $152 \%$ \\
\hline UEP7 & $101 \%$ & $119 \%$ & $122 \%$ & $117 \%$ \\
\hline UEP8 & $102 \%$ & $110 \%$ & $169 \%$ & $120 \%$ \\
\hline UEP9 & $94 \%$ & $143 \%$ & $144 \%$ & $129 \%$ \\
\hline UEP10 & $128 \%$ & $139 \%$ & $128 \%$ & $96 \%$ \\
\hline UEP11 & $121 \%$ & $141 \%$ & $106 \%$ & $173 \%$ \\
\hline UEP12 & $43 \%$ & $61 \%$ & $138 \%$ & $149 \%$ \\
\hline UEP13 & $96 \%$ & $141 \%$ & $143 \%$ & $122 \%$ \\
\hline UEP14 & $72 \%$ & $103 \%$ & $116 \%$ & $83 \%$ \\
\hline UEP15 & $73 \%$ & $100 \%$ & $119 \%$ & $127 \%$ \\
\hline UEP16 & $5 \%$ & $17 \%$ & $153 \%$ & $244 \%$ \\
\hline UEP17 & $80 \%$ & $109 \%$ & $104 \%$ & $100 \%$ \\
\hline
\end{tabular}


Indicadores de Desempenho como Ferramenta de Alinhamento Estratégico - A Experiência do Senac Paraná

\begin{tabular}{|l|c|c|c|c|}
\cline { 2 - 5 } UEP18 & $76 \%$ & $112 \%$ & $142 \%$ & $106 \%$ \\
\hline UEP19 & $136 \%$ & $147 \%$ & $141 \%$ & $146 \%$ \\
\hline UEP20 & $76 \%$ & $91 \%$ & $92 \%$ & $63 \%$ \\
\hline UEP21 & $0 \%$ & $7 \%$ & $131 \%$ & $185 \%$ \\
\hline UEP23 & $50 \%$ & $94 \%$ & $116 \%$ & $199 \%$ \\
\hline UEP24 & $5 \%$ & $97 \%$ & $113 \%$ & $112 \%$ \\
\hline UEP25 & $133 \%$ & $117 \%$ & $91 \%$ & $228 \%$ \\
\hline UEP26 & $88 \%$ & $97 \%$ & $61 \%$ & $137 \%$ \\
\hline UEP27 & $86 \%$ & $113 \%$ & $147 \%$ & $229 \%$ \\
\hline UEP28 & $30 \%$ & $28 \%$ & $35 \%$ & $92 \%$ \\
\hline UEP29 & $61 \%$ & $92 \%$ & $123 \%$ & $156 \%$ \\
\hline UEP30 & $38 \%$ & $137 \%$ & $138 \%$ & $168 \%$ \\
\hline UEP31 & $47 \%$ & $112 \%$ & $96 \%$ & $43 \%$ \\
\hline UEP32 & $142 \%$ & $149 \%$ & $161 \%$ & $237 \%$ \\
\hline UEP33 & $71 \%$ & $112 \%$ & $141 \%$ & $59 \%$ \\
\hline
\end{tabular}

Fontes: Relatórios dos sistemas gerenciais Sapiens e Orçamento Programa - ano de 2013.

Estabelecendo-se como hipótese nula (Ho) que o percentual de execução dos valores orçados de investimentos em gratuidade independe da unidade, aplica-se então a técnica estatística de análise de variância, com a mesma finalidade da análise do indicador anterior.

O resultado da análise estatística dos dados da Tabela 3 pode ser observado na Tabela 4:

Tabela 4 - Análise estatística do percentual da meta de gratuidade executada em 2013

\begin{tabular}{|l|c|c|c|c|}
\hline & $\mathbf{1}^{\mathbf{0}}$ Trimestre & $\mathbf{2}^{\mathbf{0}}$ Trimestre & $\mathbf{3}^{\mathbf{0}}$ Trimestre & $\mathbf{4}^{\mathbf{0}}$ Trimestre \\
\hline Tamanho da amostra & 33 & 33 & 33 & 33 \\
\hline Média Aritmética & 0.7970 & 1.0227 & 1.3033 & 1.3942 \\
\hline Variância & 0.2261 & 0.1409 & 0.2042 & 0.5199 \\
\hline Desvio Padrão & 0.4755 & 0.3754 & 0.4519 & 0.7211 \\
\hline Valor da estatística F: 8,9630; nível de significância: 5\%. \\
\hline
\end{tabular}

Desta forma, rejeita-se a hipótese nula $(\mathrm{Ho})$ e aponta-se para a existência de diferença estatisticamente significativa no percentual de execução do valor de investimento em gratuidade orçado, nas unidades analisadas, ao longo do ano de 2013.

Dada esta situação, os questionamentos sugeridos para os resultados do indicador "percentual da meta de gratuidade executada" são os seguintes:

- Quais as estratégias adotadas pelos gestores das unidades com melhor performance?

- Até que ponto o perfil mais arrojado ou mais conservador, de cada gestor, impacta no dimensionamento das metas?

- Como se dá o processo de incorporação de cotas extras de gratuidade? Tal incorporação está atrelada a análise da capacidade real de execução de cada unidade?

- Como está ocorrendo a oferta em unidades remotas, cedidas por instituições parceiras?
- Como se dá o processo de divulgação dos programas nestas unidades?

- Os questionamentos propostos visam tão somente exemplificar o exercício de gestão a partir dos resultados apresentados pelos indicadores, embora sejam aplicáveis na temática específica de análise deste artigo.

\section{CONSIDERAÇÕES FINAIS}

A prática exemplificada por meio deste estudo de caso não pretende trazer soluções prontas ou esgotar as análises. Pelo contrário: propõe iniciar um processo de reflexão acerca da construção e análise de indicadores de desempenho que auxiliem no processo de alinhamento estratégico.

A proposta é que as organizações, em especial as incluídas no âmbito do Sistema $\mathrm{S}$, conscientizem-se da importância de sistematizar a verificação do alinhamento das ações locais às estratégias globais, 
apropriando-se, para tanto, de ferramentas de gestão como o BSC e softwares estatísticos.

Os indicadores propostos neste estudo de caso apenas exemplificam a utilização destas ferramentas, dando margem à sua utilização em um rol infinito de indicadores, para alinhamento das mais variadas estratégias institucionais.

O processo de alinhamento estratégico tem se mostrado fundamental, dada a quantidade cada vez maior de projetos ofertados simultaneamente, nos mais variados programas, em dezenas de unidades executivas, que representa uma característica marcante das instituições do Sistema S. Esta pluralidade de ações tem exigido dos gestores a construção de mecanismos que possibilitem tanto o seu monitoramento e controle, blindando sua atuação junto aos órgãos de controle, quanto a racionalização dos recursos em prol do cumprimento da missão à qual se propõe a instituição.

No caso particular dos indicadores analisados neste artigo, sua contribuição para o alinhamento das iniciativas locais à estratégia global de promoção social traz, à sociedade, muitos benefícios. De fato, o que verifica-se em todos os municípios onde o SENAC atua de forma efetiva com os programas de gratuidade é uma gradativa evolução do panorama socioeconômico por meio da educação profissional, uma vez que esta promove inclusão produtiva, fortalecimento do comércio e aumento da renda e da qualidade de vida da comunidade. Neste sentido, a aferição e o acompanhamento dos níveis de investimentos em gratuidade em cada uma das regiões paranaenses onde o SENAC atua, promovendo o seu alinhamento estratégico, oportuniza uma aplicação mais efetiva dos recursos em prol da educação profissional inclusiva nestas regiões, com impacto direto no desenvolvimento socioeconômico de cada uma delas.

\section{REFERÊNCIAS}

Achwarz, F.; Amiden, M. A. M. \& Pinho, L. C. (2005), Alinhamento e desdobramento da estratégia. In: Kallás, D.; Coutinho, A. R. Gestão da estratégia: experiências e lições de empresas brasileiras. Rio de Janeiro: Elsevier.

Caiuby, R. D. \& Luciano, D. M. (2005), Vinculando estratégia aos processos operacionais. In: Kallás, D.; Coutinho, A. R. Gestão da estratégia: experiências e lições de empresas brasileiras. Rio de Janeiro: Elsevier.

Costa, A. P. P. (2006), Balanced Scorecard. Conceitos e guia de implementação. São Paulo: Atlas.
Damke, E. J.; Silva, E. D. \& Walter, S. A. (2011), Sistemas de controle e alinhamento estratégico: proposição de indicadores. Revista Eletrônica Estratégia \& Negócios, v.4, n.1, p.65-87.

Fernandes, B. H. R. \& Berton, L. H. (2004), Administração estratégica. Da competência empreendedora à avaliação de desempenho. Curitiba: Positivo.

Gaio, J. (2012), Método de avaliação do alinhamento estratégico entre diferentes níveis organizacionais em instituição educacional religiosa, sem fins lucrativos. Foco na qualidade dos serviços prestados no ensino superior. Dissertação de mestrado, Pontifícia Universidade Católica do Paraná, Curitiba, Paraná.

Herman, R. D. \& Renz, D. O. (1999), Theses on nonprofit organization effectiveness. Nonprofit and Voluntary Sector Quarterly, v. 28, n. 2, p. 107-126.

Johnston, R. \& Pongatichat, P. (2008), Managing the tension between performance measurement and strategy: coping strategies. International Journal of Operations \& Production Management, v. 28, n. 10, p. 941-967.

Kaplan, R. S. \& Norton, D. P. (1997), A estratégia em ação. Balanced Scorecard. Rio de Janeiro: Campus.

Kaplan, R. S. \& Norton, D. P. (2001), Organização orientada para a estratégia - The StrategicFocused Organization. Rio de Janeiro: Campus.

Lima, C. R. M.; Soares, T. C. \& Lima, M. A. (2011), Balanced scorecard em instituições de ensino superior: uma análise das perspectivas. Revista Eletrônica Estratégia \& Negócios, v.4, n.1, p.183205.

Manzione, S. (2006), Marketing para o terceiro setor. São Paulo: Novatec.

Müller, C. J.; Cortimiglia, M. N.; Gabrieli, L. V. \& Kappel, A. M. (2003), Gerenciamento de processos $e$ indicadores em educação à distância. In: Encontro Nacional De Engenharia De Produção, 23., 2003, Ouro Preto. São Paulo: ENEGEP.

Niven, P. R. (2003), Balanced Scorecard: Step-by-Step for Government and Nonprofit Agencies. John Wiley \& Sons: New Jersey.

Walter, S. A.; Tontini, G.; Andrade, K. A. \& Bach, T. M. (2014), Qualidade percebida de stakeholders como estratégia de melhoria dos serviços. Revista Eletrônica Estratégia \& Negócios, v.7, n.1, p.1-28. 
Indicadores de Desempenho como Ferramenta de Alinhamento Estratégico - A Experiência do Senac Paraná

Wunder, T. (2005), New strategy alignment in Montvale: Institute of Management Accountants. v. multinational corporations. In: Strategic Finance, 87 , n. 5. 